\title{
THREE-DIMENSIONAL IMAGE SEGMENTATION OF UPPER AIRWAY BY CONE BEAM CT: A REVIEW OF LITERATURE
}

\author{
Sara M. El Khateeb *
}

\begin{abstract}
The aim of this paper is to review the different approaches of three-dimensional image segmentation using cone-beam computed tomography (CBCT), with a focus on the human upper airway. Literature reviews in the dental field have been included, relating to the use of CBCT to assess the upper airway using image segmentation.

Obstruction of the upper airway often modifies normal breathing, which can have a noticeable impact on the typical development of craniofacial structures. CBCT is a modality that allows for the improved understanding of airway anatomy, pathology and upper airway analysis. It is more accurate, efficient and has a relatively less radiation dose compared to multi-detector CT.

Three-dimensional (3D) models of the upper airway that have been segmented and designed from $\mathrm{CBCT}$ images can be used to visualize and analyze treatment efficiency in subjects with breathing or obstruction disorders. The accuracy of these 3D morpho-functional analytical models is essential for improving diagnosis, treatment planning, and assessing treatment outcomes of the upper airway.
\end{abstract}

Therefore, the purpose of the present review is to discuss the different methods for upper airway segmentation using CBCT to achieve accurate modeling.

KEYWORDS: Upper Airway, Image, Segmentation, Cone Beam CT, Three-dimensional

\section{INTRODUCTION}

The human upper airway is a complex structure which expands from the tip of the nose to the higher part of the trachea, including the pharyngeal airway, laryngeal airway, and adjacent structures. ${ }^{(1)}$
Visualization and analysis of the upper airway dimensions have received great attention in the field of dentistry. This is because ${ }^{(2-4)}$ any obstruction in the upper airway increases upper airway resistance, which alters breathing and can have a significant effect on the normal growth of craniofacial structures.

* Assistant Professor, Department of Basic Dental Sciences, College of Dentistry, Princess Nourah bint Abdulrahman University, Riyadh, KSA. Oral Medicine, Periodontolgy, Diagnosis and Oral Radiology, Faculty of Dentistry, Ain Shams University, Cairo, Egypt 
In addition to this, the anatomical narrowing of the upper airway is recognized as the most documented risk factor for obstructive sleep apnea (OSA). ${ }^{(5)}$ These obstructions can be detected through the use of airway imaging.

The communal influence between the pharyngeal and skeletal structures is a topic of interest for both orthodontists and maxillofacial surgeons. ${ }^{(6)}$ However, no conclusion has been reached regarding the reason-and-outcome association between nasal obstruction, craniofacial morphology and occlusal characteristics. Mouth breathing affects the facial shape and is thought to be a disposing factor to the development of "long face syndrome" or "adenoid facies" ${ }^{(7)}$. On the other hand, mouth breathing is sometimes considered a para-phenomenon with the occurrence of altered facial morphology. ${ }^{(8)}$

Cone-beam CT (CBCT) is an accurate and efficient modality for imaging and assessing the human upper airway, particularly because of its lower cost and isotropic voxels with higher resolution. CBCT provides reliable and accurate linear and angular measurements, as well as having a lower radiation dose than multi-detector CT. Although there is poor discrimination between thin bony structures and soft tissues in CBCT, the borders between hollow spaces and soft tissues or bones are well distinguished. ${ }^{(9)}$

The first step in upper airway analysis is image segmentation. This enables the generation of accurate, patient-specific 3D models of the upper airway by CBCT. These models are used to visualize and investigate treatment efficacy in patients with breathing or obstruction disorders.

With this said, providing an accurate segmentation modality for morpho-functional analysis of the upper airway is essential for improving diagnosis, treatment planning, accurate designing of therapeutic devices and assessing treatment outcomes.

Therefore, the aim of this study is to review the different strategies for image segmentation of the upper airway using CBCT.

\section{LITERATURE REVIEW}

\section{Image Segmentation}

Image segmentation involves the extraction of structural data of precise attention from adjacent images for vision or characterization of the anatomy or pathology, utilizing three-dimensional reconstruction to do $\mathrm{so}^{(3)}$.

Segmentation also refers to the detachment of an image into areas with homogenous properties (such as gray level, color, texture, brightness and contrast, or a combination of all of this information) from the neighboring structures or background. ${ }^{(10,11)}$ It is one of the aims of image processing and computer applications that is considered to be among the most significant elements of clinical diagnostic tools. ${ }^{(10)}$

Segmentation allows for the identification and delineation of the anatomical structures of interest in all medical/dental images. ${ }^{(10)}$ Dental image segmentation is a challenging task due to the complexity of the images and the lack of linear features. ${ }^{(12)}$

3D segmentation of the upper airway by CBCT facilitates the examination of the anatomy and the function of narrow airways, especially in cases of sleep-disordered breathing and in ways that were inaccessible before. ${ }^{(13)}$

\section{Types of image segmentation:}

Methods of image segmentation are either classified according to gray level features ${ }^{(14)}$ or split into the following categories: manual, semiautomatic, and automatic segmentation. ${ }^{(11)}$

\section{Methods based on gray level features:}

Thresholding segmentation, edge-based segmentation and region-based segmentation. ${ }^{(10)}$

\section{Thresholding technique:}

This is one of the most basic image segmentation methods, as it is easy to implement and quick in terms of computation. Thresholding is often used 
for MR and CT angiographic studies where there is significant blood/tissue contrast, as well as for bone and upper airway segmentation in CBCT. Thresholding is classified into two types: global and local thresholding. ${ }^{(15,11)}$

\section{Global (Bimodal) thresholding:}

Global thresholding is the simplest segmentation technique. All areas under a selected pixel intensity (threshold) are recognized as air, while everything above that is recognized as tissue. The most important step in this technique is the selection of the value of threshold intensity by the operator or algorithm. ${ }^{(16)}$

This method is appropriate for an image with an area or object of even brightness which is placed against a background of diverse gray level (two different gray level values). ${ }^{(17)}$ Its main target is to categorize the pixels of a specified image into two classes: those relating to an object (foreground), and those relating to the background. This is called "bimodal thresholding". The technique is not widely used without a pre-processing algorithm due to its sensitivity to noise. ${ }^{(11)}$.

\section{Local (Multimodal) thresholding}

This method is useful when several structures are present with dissimilar gray level values. This is called "multimodal thresholding". Multi-thresholding is performed by determining three different groups, each of which is comprised of pixels with similar intensities. These groups outline the portions of the image with intensities corresponding to air, tissue and bone. ${ }^{(18)}$

Kabaliuk N. et al. (18) reported that multithresholding is unreliable for CBCT data, as noise is likely to gather in the image and cause oversegmentation. This is particularly true for bones that contain multiple inner structures, such as the nasal cavity, which can lead to extensive variation of the bone pixel intensity. As a result, this sometimes overlays the distinction of other tissue types. ${ }^{(17)}$

\section{Edge-based segmentation:}

This method segments an image based on the boundaries within it, i.e. the borders that separate discrete areas. The edge recognition method involves the marking of discontinuities in gray level, color, etc., and often these edges represent borders between objects. However, this technique is often influenced by the presence of noise and weak edges. ${ }^{(11,19)}$

\section{Region-based segmentation or region growing segmentation:}

This method revolves around the principle of homogeneousness, whereby pixels with similar features are gathered together to form a homogenous region. The homogeneity properties are differentiated by the gray value of pixels. The method begins with the operator selecting a certain pixel, which is referred to as the seed pixel. After this has been chosen, only similar pixels surrounding the seed pixel are automatically added to the region. Unfortunately, the performance of this method is impacted by the presence of noise and weak edges, leading to under- or over-segmentation of certain areas in the picture. This can be resolved by combining region-based segmentation with edge-based segmentation. ${ }^{(10,20)}$

\section{Manual, semi-automatic and automatic segmentation}

Image segmentation can also be divided into three further categories: manual, semi-automatic, and automatic. ${ }^{(11)}$

\section{Manual segmentation}

With manual segmentation, the operator carries out manual tracing or freehand drawing of the borders or grey threshold values of the same intensity level for segmentation. This is an accurate method but also very time-consuming, especially when the image is large; the operator must sketch the structure boundaries on separate cuts and then convert the data into a three-dimensional volume. 
Despite this, it does offer a precise 3D rendering of the airway. ${ }^{(21)}$

In addition to the lengthy process involved, the limitations of this technique include exposure to observer variability and expensive human resources. As a result, there is a strong demand for automating the segmentation procedure. With that said, manual segmentation is the most accurate and frequently used method; it ensures that the operator has complete control and can deliver all essential anatomical data, which is essential in certain situations. ${ }^{(22)}$

\section{Automatic segmentation}

Automatic segmentation has the advantage of drastically reducing segmentation time. Most algorithms depend on some prior information (preset data) to carry out the segmentation, and this information must be accessible by the computer. ${ }^{(21)}$ The required prior data could be, for example, noise level and the probable special distribution of the objects. For low contrast images, some of these algorithms deliver suboptimal explanations which are not clinically acceptable. ${ }^{(21)}$

Many commercial software products offer automatic segmentation. Although this is time effective, it is not as precise as manual segmentation. These software programs tend to "combine" the gray threshold values of the whole area of interest, rather than customizing them based on location. ${ }^{(23)}$

\section{Semi-automatic segmentation:}

This segmentation method combines the advantages of both manual and automatic segmentation. Some initial data about the structures are supplied by the operator and this is followed by automatic methods. ${ }^{(21)}$

\section{Applications of image segmentation}

The most common applications of image segmentation include 3D visualization of detailed patient anatomy, volumetric measurements, image- guided surgery to supply surgeons with a 3D model of patient-specific anatomy (thus avoiding hazards such as blood vessels, nerves and vital structure injuries), and detection of anatomical changes over time. ${ }^{(24)}$

\section{General limitations of upper airway segmentations}

\section{Thresholding:}

The appropriate selection of threshold values is relatively difficult, as it is affected by factors such as volume averaging. Consequently, the CBCT values of miniscule bony walls will drop below the thresholding range of bone because their density is close to that of surrounding air. This results in false holes in three dimensional reconstructions. ${ }^{(25)}$ Noise caused by metal artifacts (such as orthodontic appliances, dental fillings, implants, surgical plates, etc.) also affects performance, with metal error intensity levels dropping into the thresholding range of bone and appearing in CBCT images as pronounced, "star-like" streaks. ${ }^{(10,20)}$

As the nasal cavity has several complicated, narrow passageways and adjacent paranasal sinuses, it is particularly vulnerable to either undersegmentation (true borders between tissue and air are unnoticed by the segmentation algorithm, and discrete air-filled holes are fused into one) or oversegmentation (the algorithm yields false borders which are not anatomically present) ${ }^{(18)}$.

Therefore, the range of the gray threshold should not be fixed, neither for the pharyngeal airway nor for specific software, because the pixel grey value depends not only on the tissue contrast but also on additional factors. These include: the type of CBCT machine being used; the scanning parameters, which control the amount of radiation and signal; radiation scattering; metal error; patient movement, and machine calibration ${ }^{(12)}$. These factors will affect the pixel value, and so it is impracticable to standardize an airway threshold. ${ }^{(26-28)}$ 


\section{Airway geometry:}

The complexity of upper airway geometry means that both size and morphology parameters should be included in the assessment of the airway in order for the image to be accurate. As previous studies have reported, CBCT-generated 3D models of the upper airway are similar in volume but vary significantly in shape depending on whether the airway is normal, or whether it belongs to a patient with obstructive sleep apnea. ${ }^{(29)}$ Consequently, we cannot depend on single linear measurements to analyze the upper airway using CBCT, and volume alone does not represent its shape. ${ }^{(2)}$

\section{Neck and tongue mispositioning:}

Studies by Muto T et al. 2002 and Alsufyani NA et al. $2014^{(30,31)}$ found that the effect of neck flexion during CBCT scanning can cause inconsistencies of more than $3 \mathrm{~mm}$ inter-distance at $\mathrm{C} 2-\mathrm{C} 3$, to larger discrepancies of up to around $5 \mathrm{~mm}$ between local portions of the airway models over time. Corresponding variations in volume, surface area, and point-based analysis were initiated in patients with tongue malpositioning. This effect occurs because the pharyngeal airway consists mainly of fat and muscle, which are susceptible to distortion due to compression from the cervical vertebrae posteriorly, or the tongue anteriorly. ${ }^{(2)}$

Patient movement, swallowing, breathing, neck flexion, and tongue mispositioning during CBCT scanning are all unavoidable, especially in young patients. Consequently, new approaches to stabilizing the head and neck and regulate the tongue position throughout the scan need to be established. Until then, upper airway fluctuations with tongue or neck mispositioning, particularly in the oropharyngeal airway, must be evaluated with particular attention. ${ }^{(2)}$

\section{DISCUSSION}

The anatomical structure and functional properties of the upper airway influence the development of the craniofacial complex considerably. ${ }^{(32)}$

It has also been claimed that airway function affects facial morphology over a long period of time. Although there is still some controversy around this issue, nasopharyngeal impairment is mostly present in patients with increased anterior face height. This can adversely impact dental function and esthetics $^{(33,34)}$.

In recent times, common facial orthopedic treatments have demonstrated success for pediatric $\mathrm{OSA}^{(2)}$. Subsequently, the relationship between upper airway anatomy and sleep disordered breathing development and treatment remains to be a field of interest for further research.

Lately, CBCT has been endorsed as an innovative tool that can be used to assess the upper airway. Its popularity can be attributed to its lower radiation dose, high accuracy and wide-spread availability. Furthermore, post-processing of DICOM images by third party software permits visualization, segmentation and quantification of the airway in an exclusive way. With this comes a better understanding of the anatomy and physiology of the upper airway. Drawbacks to CBCT include its suboptimal resolution due to scatter radiation, shortage of soft tissue delineation, and damaging ionizing radiation if the procedure is not properly modified to fit the needs of each patient. ${ }^{(2)}$

CBCT is being used increasingly in the linear and volumetric assessment of the upper airway, the aim of which is to evaluate the effects of various orthodontic and surgical treatments. It is especially useful in cases of obstructive sleep apnea (OSA) in both children and adults. ${ }^{(35,36,37)}$ Despite this, the CBCT protocol for airway imaging remains vague. (38) The first step in CBCT airway analysis is to review the different approaches to segmenting CBCT images in order to formulate 3D modeling and volumetric measurements. 
Image segmentation is mandatory for generating an accurate, patient-specific 3D model of the upper airway. Automatic and semi-automatic image segmentation methods are more applicable than manual image segmentation, which is very time consuming but more accurate. On the other hand, the automatic segmentation of the upper airway, especially when the nasal cavity and paranasal sinuses are involved, can be very challenging. This is due to noise, artifacts $^{(39)}$ and the complicated anatomical structure of multiple thin bony structures and tortuous air passages. Recently, many studies have reported variable automatic and semi-automatic methods for image segmentation of the human upper airway using CBCT. ${ }^{(38)}$

Most studies only analyze the pharyngeal airway with volumetric measurements, while Iannetti et al. ${ }^{(40)}$, Iwasaki et al. ${ }^{(41)}$ and $\mathrm{El}$ and Palomo ${ }^{(42)}$ segmented the nasal cavity and maxillary sinuses. The shape of the oropharyngeal airway is tube-like, and it is completely hollow, making the process of segmentation straightforward. The anatomy of the nasal cavity, on the other hand, is complicated, with the narrow and twisting pathways of the conchae and meatuses. Consequently, the segmentation process is extremely challenging due to difficulties with defining boundaries and grey thresholding, especially with noisy CBCT images. Therefore, studies that only focus on the oropharyngeal airway will likely over-represent the true validity of the assessed tools. ${ }^{(39)}$ Assessments looking at the accuracy of segmentation that are only based on studies of the oropharyngeal airway are not directly applicable to segmentations of the whole airway. ${ }^{(39)}$

Leading on from this, Nhat Linh Bui et al.'s 2015 study included the nasal cavity and paranasal sinuses. They offered a multi-step scheme for the automated segmentation and reconstruction from CBCT images, using a coarse-to-fine active contour model ${ }^{(43)}$ to create a 3D model of the nasal cavity and paranasal sinuses. The accurateness of the planned scheme was greater than present CBCT segmentation methods, with global and localized region-based active contouring, and there were few cases where imprecise segmentation occurred.

Although 3D imaging of the upper airway is very effective for volumetric airway measurements and detection of any obstructions, it only produces a quick shot of the head during sleep - it fails to reflect the complexity of airway function in the field of sleeping disorders. ${ }^{(2)}$.

Alsufyani et al. $2012^{(38)}$ reported the lack of a reliable and optimized CBCT protocol for airway imaging. They also noted the limited number of published studies that assess the validity and reliability of segmented 3D airway models generated from CBCT images, through either applied automatic or semi-automatic techniques. In fact, only $50 \%$ of the reviewed studies attempted to validate the segmentation results and describe the methodology. Alsufyani et al. ${ }^{(38)}$ stated that the accuracy and versatility of the few existing automatic and semiautomatic methods are under-researched.

CBCT used to produce 3D airway replica which are presented as objective assessment methods in the surgical treatment of OSA patients, orthognathic surgery, maxillary expansion and general impression on airway measurements. For this reason, it is necessary to scientifically validate the technique used to create these replicas, as they act as an essential baseline for upper airway treatment. ${ }^{(2)}$

\section{CONCLUSION}

According to the literature, there is no universally accepted method for image segmentation. This is because it is affected by several different factors, such as homogeneity of images, spatial characteristics of the image continuity, texture, and image content. Thus, there is no single method that can be considered the 'best' for all image types, nor for a particular type of image. Subsequently, image segmentation remains a challenging problem in image processing and computer vision ${ }^{(44)}$, despite the fact that it is considered the preliminary stage of almost all image analyzing tools. ${ }^{(12)}$ 
As this review has demonstrated, the existing literature lacks proper scientific justification of a solid and adjusted CBCT protocol for upper airway segmentation. Due to the limited number of adequate studies, it is difficult to generate a strong conclusion regarding the validity and reliability of CBCTgenerated 3D models. Most 3D airway models generated from CBCT have not been authenticated in the literature.

\section{Future recommendations}

Future research should be designed to consider and address the following:

1. Conducting an extensive longitudinal study to develop upper airway norms that determine the typical values for upper airway volume and other linear measurements. These should be relevant to specific age groups and genders and should be taken from individuals who have normally developed upper airways. These values can then be used as baseline measurements, which can be compared to the values obtained from afflicted individuals.

Moreover, these norms can be utilized as the gold standard to validate measurements obtained by different segmentation methods, replacing the currently used benchmark values.

2. Innovations in standardizing and stabilizing the patient's neck and tongue during the scan through the creation of policies and/or appliances.

3. It would be beneficial for future research to divide the upper airway into both nasal and pharyngeal parts for analysis.

\section{REFERENCES}

1. Bui NL, Ong SH, Foong KW. Automatic segmentation of the nasal cavity and paranasal sinuses from cone-beam CT images. International journal of computer assisted radiology and surgery. 2015 Aug 1;10(8):1269-77.

2. Alsufyani NA, Noga ML, Witmans M, Major PW. Upper airway imaging in sleep-disordered breathing: role of cone-beam computed tomography. Oral Radiology. 2017 Sep 1;33(3):161-9.

3. Abramson Z, Susarla SM, Lawler M, Bouchard C, Troulis M, Kaban LB. Three-dimensional computed tomographic airway analysis of patients with obstructive sleep apnea treated by maxilla-mandibular advancement. J Oral Maxillofac Surg 2011; 69: 677-686.

4. McCrillis JM, Haskell J, Haskell BS, Brammer M, Chenin D, Scarfe WC, et al. Obstructive sleep apnea and the use of cone beam computed tomography in airway imaging: A review. Semin Orthod 2009; 15: 63-69.

5. Masumi S, Clark G.; Effect of jaw position and Posture On forced Inspiration Airflow. Chart 109:1477- 1483, 1996.

6. Martin O, Muelas L, Vinas MJ. Nasopharyngeal cephalometric study of ideal occlusions. Am J Orthod Dentofacial Orthop. 2006;130(4): 436.e1-9. DOI: 10.1016/j. ajodo.2006.03.022

7. Bresolin D, Shapiro PA, Shapiro GG, Chapko MK, Dassel S. Mouth breathing in allergic children: its relationship to dentofacial development. Am J Orthod. 1983; 83(4):334340. DOI:10.1016/0002-9416(83)90229-4

8. Emslie RD, Massler M, Zwemer JD. Mouth breathing. Etiology and effects; a review. J Am Dent Assoc (1939). 1952; 44(5):506-521. DOI: 10.14219/jada.archive. 1952.0099

9. Guijarro-Martinez R, Swennen GR: Cone-beam computerized tomography imaging and analysis of the upper airway: a systematic review of the literature. Int J Oral Maxillofac Surg, 2011, 40 (11):1227-123.

10. Sharma N, Aggarwal LM. Automated medical image segmentation techniques. J Med Phys. 2010;35(1):3-14.

11. Khalifa I YA, Youssry H. Tissue segmentation Techniques of brain MR images: A Review. International Conference on Intelligent Computational Systems (ICICS'2012). Dubai; 2012

12. Balafar MA, Ramli AR, Saripan MI, Mashohor S. Review of brain MRI image segmentation methods. Artificial Intelligence Review. 2010;33(3):261-274.

13. Hatcher DC. Cone beam computed tomography: Craniofacial and airway analysis. Sleep Medicine Clinics. 2010; 5:59-70.

14. Withey DJ KZ. Three generations of medical image segmentation: Methods and available software. Int J Bioelectromag 2007;.9:67-68. 
15. Tucker SF. Orthognathic Surgical Simulation Of Class Iii Patients Using 3-D Cone Beam Ct Images. Orthodontics University of North Carolina at Chapel Hill 2009.

16. Bankman, I.N. (2000) The Handbook of Medical Imaging: Processing and Analysis. Academic Press.

17. Ramesh N YJ, Sethi IK. Thresholding based on histogram approximation. IEEE Proc Vision Image Signal Proc 1995; 142:271-279.

18. Kabaliuk N, Nejati A, Loch C, Schwass D, Cater JE, Jermy MC. Strategies for Segmenting the Upper Airway in ConeBeam Computed Tomography (CBCT) Data. Open Journal of Medical Imaging. 2017 Sep 29;7(04):196.

19. Withey DJ, Koles, Z.J. Medical Image Segmentation: Methods and Software. Hangzhou: Noninvasive Functional Source Imaging of the Brain and Heart and the International Conference on Functional Biomedical Imaging, 6th International Symposium 140 - 143,2007.

20. Sharma N RA. Computer aided segmentation of medical images based on hybridized approach of edge and regionbased techniques. Proc. of Int. Conf. on Mathematical Biology', Mathematical Biology recent trends by ANAMAYA Publishers; 2006:150-155.

21. Hodneland E. Segmentation of digital images. Cand. scient thesis, Department of Mathematics, University of Bergen. 2003.

22. Calder J, Tahmasebi AM, Mansouri A-R. A variational approach to bone segmentation in CT images. Paper presented at: SPIE Medical Imaging, 2011.

23. Alsufyani NA: Evaluation of anatomic surgical outcomes in children with sleep disordered breathing symptoms using Cone Beam computed tomography, (unpublished Ph.D. dissertation), Alberta, Canada, University of Alberta, 2015.

24. O’Donnell L. Semi-Automatic Medical Image Segmentation: Massachusetts Institute of Technology master thesis in Computer Science and Engineering 2001.

25. Maillet M, Bowles WR, McClanahan SL, John MT, Ahmad M. Cone-beam computed tomography evaluation of maxillary sinusitis. J Endod. 2011;37(6):753-757.

26. Schulze R, Heil U, Gross D, Bruellmann DD, Dranischnikow E, Schwanecke U, et al. Artefacts in CBCT: a review. Dentomaxillofac Radiol. 2011; 40:265-73.

27. Seo A, Chung SK, Lee J, Kim JI, Kim H. Semiautomatic segmentation of nasal airway based on collaborative envi- ronment. Proceedings of the International Symposium on Ubiquitous Virtual Reality ISUVR; 2010 July 7-10; Washington, DC, USA.

28. Tingelhoff K, Moral AI, Kunkel ME, Rilk M, Wagner I, Eichhorn KWG, et al. Comparison between manual and semi-automatic segmentation of nasal cavity and paranasal sinuses from CT images. Proceedings of the Annual International Conference of the IEEE Engineering in Medicine and Biology; 2007 August 23-26; Lyon, France. doi:10.1109/IEMBS.2007.4353592.

29. Alsufyani NA, Al-Saleh MA, Major PW. CBCT assessment of upper airway changes and treatment outcomes of obstructive sleep apnea: a systematic review. Sleep Breath. 2013; 17:911-23.

30. Muto T, Takeda S, Kanazawa M, Yamazaki A, Fujiwara Y, Mizoguchi: The effect of head posture on the pharyngeal airway space (PAS). Int J Oral Maxillofac Surg. 2002; 31:579-583.

31. Alsufyani NA, Dietrich NH, Lagravère MO, Carey JP, Major PW. Cone beam computed tomography registration for 3-D airway analysis based on anatomic landmarks. Oral Surg Oral Med Oral Pathol Oral Radiol. 2014; 118:371-383.

32. Lenza MG, Lenza MD, Dalstra M, Melsen B, Cattaneo PM. An analysis of different approaches to the assessment of upper airway morphology: a CBCT study. Orthodontics \& craniofacial research. 2010 May;13(2):96-105.

33. Osorio F, Perilla M, Doyle DJ, Palomo JM. Cone beam computed tomography: an innovative tool for airway assessment. Anesth Analg 2008; 106: 1803-7.

34. Solow B, Siersback-Nielsen S, Greve E. Airway adequacy, head posture, and craniofacial morphology. Am J Orthod 1984; 86: 214- 23.

35. Katyal V., Pamula Y., Martin A.J., Daynes C.N., Kennedy J.D. and Sampson W.J. Craniofacial and upper airway morphology in pediatric sleep-disordered breathing: Systematic review and meta-analysis. American Journal of Orthodontics and Dentofacial Orthopedics, 2013, 143, 20-30.

36. Huynh N.T., Desplats E. and Almeida F.R. Orthodontics treatments for managing obstructive sleep apnea syndrome in children: a systematic review and meta-analysis. Sleep Medicine Reviews, 2016, 25, 84-94.

37. J. N. Zimmerman, Janson Lee, and Benjamin T. Pliska. Reliability of upper pharyngeal airway assessment using 
dental CBCT: a systematic review, European Journal of Orthodontics, 2017, Vol. 39, No. 5)

38. Alsufyani N.A., Flores-Mir C. and Major P.W.: Threedimensional segmentation of the upper airway using cone beam CT: a systematic review. Dento Maxillo Facial Radiology, 2012, 41, 276-284.

39. Katsumata A, Hirukawa A, Noujeim M, Okumura S, Naitoh M, Fujishita M, Ariji E, Langlais RP: Image artifact in dental cone-beam CT. Oral Surg OralMed Oral Pathol Oral Radiol Endod, 2006, 101:652-657.

40. Iannetti G, Polimeni A, Pagnoni M, et al. Upper airway volume after le fort III advancement in subjects with craniofacial malformation. J Craniofac Surg. 2011; 22:351-5.

41. Iwasaki T, Saitoh I, Takemoto Y, et al. Evaluation of upper airway obstruction in class II children with fluid-mechanical simulation. American Journal of Orthodontics and Dentofacial Orthopedics. 2011;139: e135-e145.

42. El H, Palomo JM. Measuring the airway in 3 dimensions: A reliability and accuracy study. American Journal of Orthodontics and Dentofacial Orthopedics. 2010;137: S50. e1-S50.e9.

43. Linh Bui · Sim Heng Ong · Kelvin Weng Chiong Foong. Automatic segmentation of the nasal cavity and paranasal sinuses from cone-beam CT images Nhat Int J CARS (2015) 10:1269-1277

44. Yang D, Zheng J, Nofal A, Deasy J, El Naqa IM. Techniques and software tool for 3D multimodality medical image segmentation. Journal of Radiation Oncology Informatics. 2009;1(1):1-22. 